\title{
INFLUENCE OF X DIRECTION MOTION OF THE SUSPENSION POINT AND RIGID BODY SWING ON THE DISPLACEMENT OF A STEEL CATENARY RISER UNDER WAVE ACTION
}

\author{
ZHU, B. ${ }^{*}{ }^{*}$ HUANG, W. P. ${ }^{1}-$ YAO, X. L. ${ }^{1}-$ LIU, J. ${ }^{2}$ \\ ${ }^{I}$ Shangdong Province Key Laboratory of Ocean Engineering, Ocean University of China, \\ Qingdao 266071, China \\ ${ }^{2}$ Institute of Civil Engineering, Agriculture University of Qingdao, Qingdao 266009, China \\ *Corresponding author \\ e-mail: beiji_dongjie@qq.com; phone:+86-176-8574-3056
}

(Received $12^{\text {th }}$ Feb 2019; accepted $10^{\text {th }}$ Apr 2019)

\begin{abstract}
The response and force effect of rigid body swing (RBS) on transverse flow direction is not negligible. Based on slender beam model, wave action (WA), RBS model and in-line movement of suspension point (TSM) are simulated. And structural response effect of RBS is simulated and estimated. The simulation and evaluation display that in terms of overall motion pattern, response in xy plane develops gradually from a narrow swing in $\mathrm{x}$ direction to a narrow swing in y direction. It is important to consider a particular location conservation of energy in three directions with water depth. As water depth increases, displacement and main frequency decrease. The decrease amplitude is strongest or most severe in top suspension region. For the spectrum analysis, the response main frequency and wave frequency are very close, and the motion is a forced motion. As water depth increases, the main frequency first increases then decreases. The trend is similarly to corresponding response change and contrary to energy or amplitude of xy plane change. As the energy decreases and then increases in xy plane, the $\mathrm{z}$ direction increase of RBS presents the opposite change in three directions. The effect of RBS on structural response can reach $20 \%$ after simulation. The effect of RBS is different from that of WA and TSM, which decreases with depth increase. And RBS is positively correlated to vector diameter S. It is expected that analysis and study may provide some advice for simulation of SCR and its RBS.
\end{abstract}

Keywords: SCR, rigid body swing, wave action, platform motion, spectrum analysis

\section{Introduction}

Steel catenary riser (SCR) is one of important facility for oil exploitation. It has recently been researched extensively and in-depth on the dynamic and vibration characteristics. And in particular the phenomenon of RBS is important and affected in the deep-sea environment. It is due to the waves, currents and other large range of loads, and it has attracted the attention.

The phenomenon of RBS is a large range and whole rotation from moving suspension point in top region to touch down point (TDP) in the seabed region. This paper focuses on the influence that WA and TSM are in the same in-line (x) direction. And RBS is in cross flow (z) direction. These motions are mutually perpendicular in the plane. A simulation was realized on the basis of Cable3D (Bai, 2009; Chen, 2002). Liu (2013), Liu and Huang (2014a, 2014b, 2013) proposed and simulated the phenomenon of RBS. And the simulation was on the basis of vibration theory of large-deflection beam and RBS model. Liu and Huang (2014c) compare analysis of coupling between RBS and bending vibration to that without RBS. They concluded that RBS has a relatively great impact on transverse flow direction response. Yao et al. (2018) did the model analysis of RBS and then carried out the spectral analysis. And he believed 
experiment could prove the existence of RBS and give the frequency formula. Yao et al. (2017) simulated RBS phenomenon coupling the vortex-induced vibration model. And he proposed a RBS model considering vortex-induced vibration. Lin and Wang (2019) adopted DVM code in 2 dimensions for riser VIV analysis. Kim and O'Reilly (2019) provided a research on the dynamic, stability and vibration of riser with Kirchhoff's theory. Lotsberg (2019) did an overview of fatigue design standards. Wu et al. (2019) provided a relatively reliable combined IL and CF load model. Yamamoto and Morooka (2019) carried out simulation for riser and platform with nonlinear models. Guan et al. (2019) obtained understanding of the mechanics of scour such as steel catenary riser without currents and waves. He et al. (2019) adopted beam bending model of large deformation characteristics and estimated the critical factors for flexible riser. Numkam et al. (2019) researched the drilling muds which display liquid and solid flow characteristics. Zhao and Van der Heijden (2019) solved the wave equation that governs the prebulking torision dynamics and boundary conditions. Wang and Pei (2019) applied a structural strain model to determine elastic characteristic of pipe core and pseudo parameters of stress.

RBS is a kind of rotation, which has rotation axis, angular velocity, angular acceleration, etc. It is different from the physical quantities such as vibration velocity and mode of bending vibration. And RBS is a rotation movement normal to bending vibration plane of riser. In the article, the effect of RBS on riser response is simulated and analyzed. And the effect of in-line (x) direction TSM and WA are studied.

The purpose of the study is in order to better discuss the RBS effect on cross flow displacement. Discussion includes the following viewpoints.

1. The RBS's effect on cross flow response cannot be ignored. And it is indispensable to pay more attention to long-term load terms and need more efforts for simulation and check.

2. Structural RBS is an inherent phenomenon of structures. And the calculation program explains RBS more from influence of WA and suspension movements.

3. The analysis of three-dimensional motion and energy conservation is very important for RBS analysis.

4. In particular, it is essential to understand characteristics of RBS when external energy of system decreases, vibration intensity of structure increases. Especially for wave reduction in this paper, RBS response increases.

5. When wave and natural vibration are in the same direction, the response is similar to that of shallow water. From top to bottom, the dominant influence of natural vibration on the structure is constantly enhanced.

6. The contribution of different loads to structural response can be calculated from frequency amplitude.

\section{Study methods}

\section{SCR beam model considering large deformation}

\section{Basic equations}

Vibration control equation (Liu, 2013) of steel catenary riser is Equation 1:

$$
M \ddot{r}+B r^{(4)}-\left(\lambda r^{\prime}\right)^{\prime}=q
$$


$M$ - mass matrix, $q$ - load matrix and $\lambda$ - Lagrange operator.

\section{SCR RBS model}

Figure 1 shows RBS model. Point A is the connection location of wellhead. Point B is TDP. Top suspension point (TSP) is Point O. $\omega$ is rotation axis vector of ODB. S is vector from rotation axis to point C (Liu and Huang, 2013; Liang et al., 2016; Du, 2016 ; Yao et al., 2018).

According to kinetic moment theorem, the equation is:

$$
\left(m+m_{a}\right) s^{2} \ddot{a}_{r}+c_{a} s^{2} \dot{a}_{r}+m g c_{1} s a_{r}=q_{z} \sqrt{s_{1}^{2}+s_{2}^{2}}+q_{x} c_{2} s_{3}
$$

$m, m_{a}$ - mass and additional mass, $c_{a}$ - coefficient for additional damping. $q_{x}, q_{z}-$ distribution load of environment, $\ddot{a}_{r}, \dot{a}_{r}, a_{r}$ - angular acceleration, velocity and displacement.

Node location:

Node 10th, $x=-15.8273 \mathrm{~m}, \mathrm{y}=-54.1376 \mathrm{~m}$.

Node 80th, $\mathrm{x}=-163.2674 \mathrm{~m}, \mathrm{y}=-467.0530 \mathrm{~m}$.

Node140th, $x=-343.3629 \mathrm{~m}, \mathrm{y}=-796.3470 \mathrm{~m}$.

Node200th, $\mathrm{x}=-616.8364 \mathrm{~m}, \mathrm{y}=-1049.0293 \mathrm{~m}$.

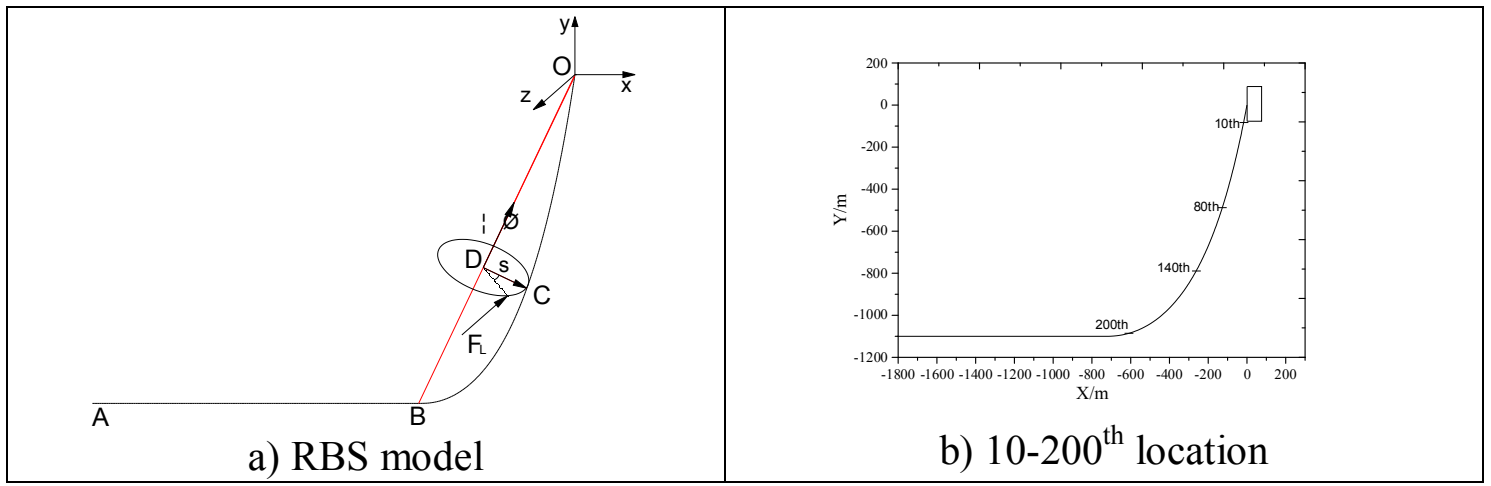

Figure 1. SCR RBS system

\section{Wave force simulation model}

Morison formula for wave force is Equation 3:

$$
f_{H}=\frac{1}{2} C_{D} \rho A\left(u_{x}-\dot{x}\right)\left|u_{x}-\dot{x}\right|+C_{M} \rho \frac{\pi D^{2}}{4} \frac{\partial u_{X}}{\partial t}-C_{m} \rho \frac{\pi D^{2}}{4} \ddot{x}
$$

In the formula, $A$ - projected area, $\rho$ - density of seawater, $C_{m}-$ coefficient of additional mass, $C_{M}$ - coefficient of mass, $C_{D}$ - coefficient of drag force, $u_{x}$ - water horizontal velocity, $\dot{x}$-cylinder horizontal velocity.

Adding Equations 2 and 3 to Equation 1, we can get: 


$$
M \ddot{r}+B r^{(4)}-\left(\lambda r^{\prime}\right)^{\prime}=q+m g-\left(m+m_{a}\right) \ddot{r}_{r}-c_{a} \dot{r}_{r}+f_{H}+Q_{x}
$$

\section{SCR movement boundary conditions}

Fixed constraints are adopted for wellhead and TSP of SCR. And TSM affects riser's whole movement. During simulation and analysis, the motion in the in-line (x) direction is added into a calculation input file. And the effect of RBS is superimposed through a load term. Then variation characteristics of the structural amplitude and frequency are studied under WA, RBS. and TSM.

\section{SCR seabed boundary conditions}

In addition to both ends fixed constraints of SCR, there are also constraints during streamline and seabed coupling interaction. It includes normal elastic and plastic support, seabed suction, tangential friction and resistance of grooves (Liu, 2013; Liu and Huang, 2014a; Yang, 2014; Zhou et al., 2017). Seabed normal constraints are simulated by spring and spring-damping system during the static and dynamic analysis. Since this part is not the focus of paper, it is not expanded in detail.

\section{Results and discussion}

\section{Response numerical simulation of RBS under linear TSM and WA}

RBS (Liu and Huang, 2014a) is an important part of SCR response study. Simulation superimposed $\mathrm{x}$ direction WA and TSM (Du, 2016) is rarely recorded in domestic and foreign literature. This section expects to analyze and summarize simulation characteristics under action of RBS, WA and TSM. It is expected to obtain some new understandings of RBS under influence of complex motion.

In this part, Cable3D and Cable3D_Vswing newly developed program (Yao et al., 2018) are adopted during numerical simulation respectively. The program of Cable3D_Vswing has a promotion of original program through Qforce subroutine. It couples and improves vortex-induced vibration, RBS and load model. It also considers the fact that wave and structure move relative to each other.

Cable3D calculates the structure's response of $\mathrm{x}$ direction WA and TSM without taking into account the action of water flow and its fluid-solid coupling. Cable3D Vswing is adopted to simulate response under effect of WA, TSM and RBS. The difference is whether RBS is taken into account during simulation.

In this paper, SCR works at a depth of $1100 \mathrm{~m}$ and is subjected to a pretension of $2100 \mathrm{KN}$. Other specific SCR parameters are shown in Table 1. The parameters of linear wave height is $3.5 \mathrm{~m}$, and cycle is $8.60 \mathrm{~s}$. WA Frequency is $0.11622 \mathrm{~Hz}$ and it is relative to $\mathrm{x}$ axis for $6.67^{\circ}$.

Table 1. SCR key parameters. (Yao et al., 2018)

\begin{tabular}{c|c|c|c}
\hline Parameters & Values & Parameters & Values \\
\hline Fluid density in the riser $\left(\mathrm{kg} \cdot \mathrm{m}^{-3}\right)$ & 865 & Elasticity Modulus $(\mathrm{Gpa})$ & 207 \\
SCR material density $\left(\mathrm{kg} \cdot \mathrm{m}^{-3}\right)$ & 7850 & Coefficient of Lift force & 0.7 \\
Seawater density $\left(\mathrm{kg} \cdot \mathrm{m}^{-3}\right)$ & 1025 & Coefficient of drag force & 1.2 \\
Outer diameter $(\mathrm{m})$ & 0.355 & Quality factor & 1.0 \\
Inner diameter $(\mathrm{m})$ & 0.305 & Hydrodynamic parameter $(\mathrm{m})$ & 0.355 \\
\hline
\end{tabular}


Coordinate system: X (in-line flow), Y (heave or vertical) and Z (cross flow). This paper applies TSM of the SCR to simulate floating platform response. Table 2 shows TSM parameters under different conditions.

Table 2. Motion parameters of different conditions. (Yao et al., 2018)

\begin{tabular}{c|c|c|c|c|c|c}
\hline Conditions & Direction & Amplitude /m & Period /s & Frequency /Hz & Cab & Cs $\boldsymbol{w}$ \\
\hline 1 & $x$ & 3.00 & 10.8 & 0.093 & $\sqrt{ }$ & $\sqrt{ }$ \\
2 & $x$ & 2.00 & 9.90 & 0.101 & $\sqrt{ }$ & $\sqrt{ }$ \\
3 & $x$ & 1.00 & 9.00 & 0.111 & $\sqrt{ }$ & $\sqrt{ }$ \\
\hline
\end{tabular}

\section{Three-dimensional response diagram of particle displacement of the structure}

The structure is subjected to WA, TSM and RBS. The node 10th-200th displacement of riser could be got through simulation. 10th-200th node displacements have been plotted for three-dimensional view with no RBS and with RBS in Figure 2. And an xy plan with RBS and no RBS is in Figure 3.

\begin{tabular}{|c|c|c|c|}
\hline $\begin{array}{l}\text { a) } 10^{\text {th }} \text { no RBS } \\
\text { displacement }\end{array}$ & $\begin{array}{l}\text { b) } 80^{\text {th }} \text { no RBS } \\
\text { displacement }\end{array}$ & $\begin{array}{l}\text { c) } 140^{\text {th }} \text { no RBS } \\
\text { displacement }\end{array}$ & $\begin{array}{l}\text { d) } 200^{\text {th }} \text { no RBS } \\
\text { displacement }\end{array}$ \\
\hline $\begin{array}{l}\text { e) } 10^{\text {th }} \mathrm{RBS} \\
\text { displacement }\end{array}$ & $\begin{array}{l}\text { f) } 80^{\text {th }} \mathrm{RBS} \\
\text { displacement }\end{array}$ & $\begin{array}{l}\text { g) } 140^{\text {th }} \mathrm{RBS} \\
\text { displacement }\end{array}$ & $\begin{array}{l}\text { h) } 200^{\text {th }} \mathrm{RBS} \\
\text { displacement }\end{array}$ \\
\hline
\end{tabular}

Figure 2. 10-200th displacement response of RBS and no RBS

In terms of transverse comparison, Figure 2 is a three-dimensional graph of response with or without RBS under TSM and WA. There is no obvious difference between two groups of graphs in morphology or overall graph. It indicates that effect of RBS is weak, which can be proved by graph with or no RBS in xy plane in Figure 3. The xy plane is the projection of structural response on the plane with $\mathrm{x}$-direction (in-line) and $y$-direction (vertical) axis. There is no important difference between two graphs in plane with in-line direction and heave direction axis. It means that there is no significant difference in the response of the bending plane of the SCR. And the effect of RBS on this plane is feeble. It can be acknowledged from a perspective of orthogonality of RBS and bending vibration. And RBS is planar motions perpendicular to bending vibrations. There is no obvious difference in the xy plane. However, the difference in $\mathrm{z}$ direction is the difference of the amplitude and curve form of the structural response. And graph and text are described in detail in the following chapters and are not expanded here. 


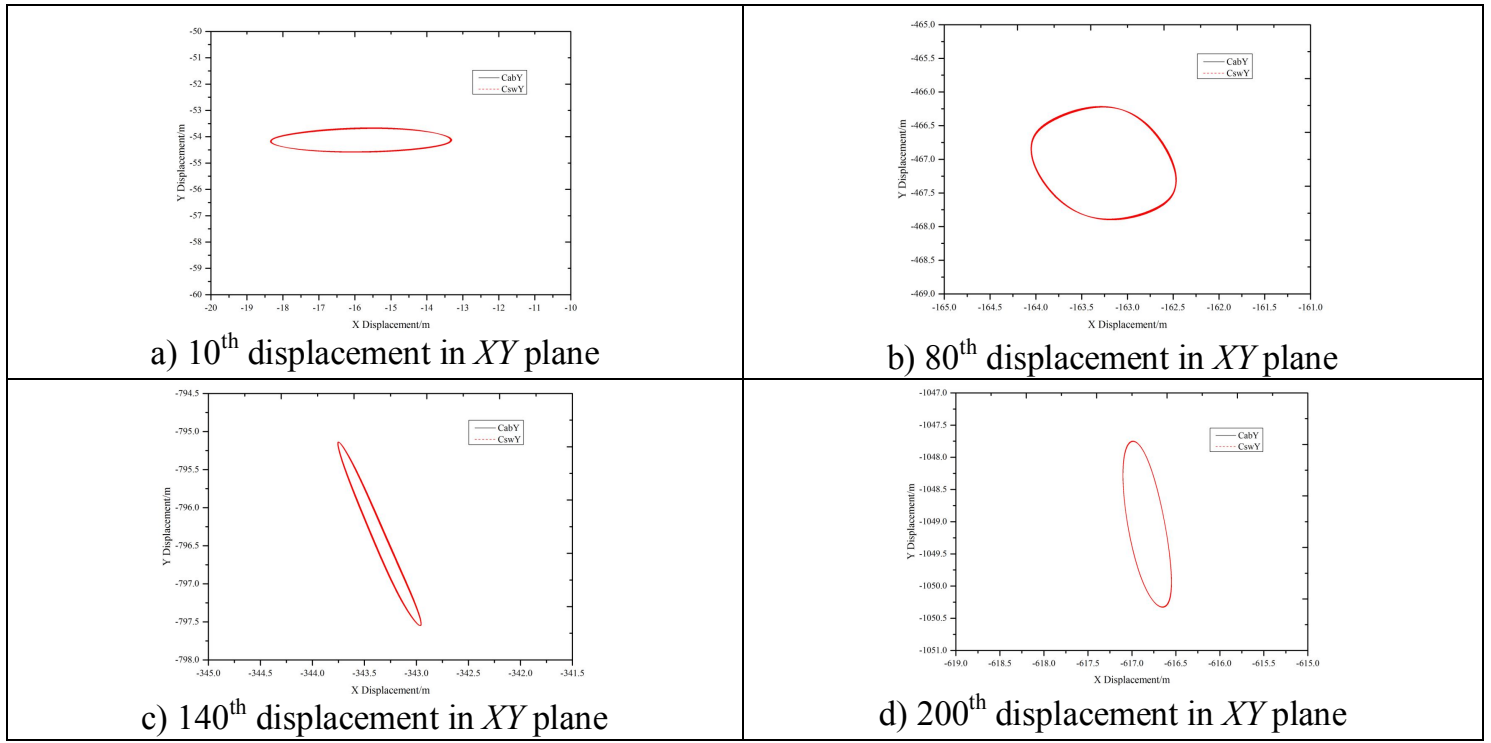

Figure 3. 10-200th displacement of RBS and no RBS in XY plane

For a longitudinal comparison, Figure 2 corresponds to node 10th-200th displacement responses, respectively. It is more appropriate to understand Figure 2 from the two-dimensional plane in Figure 3. With the increase of water depth, response on xy plane changed from a narrower swing in $\mathrm{x}$ direction to a narrower oscillation in $\mathrm{y}$ direction. The specific forms of swing are: the oscillation of 10th is a narrow range of swing in $\mathrm{x}$ direction. The oscillation of 80 th is a wide range of oscillation about $40^{\circ}$ deflection $x$ direction. The oscillation of 140th is a narrow range of oscillation around $70^{\circ}$ deflection $\mathrm{x}$ direction. The oscillation of 200th is a narrow range of oscillation around $80^{\circ}$ deflection $\mathrm{x}$ direction.

For vibration amplitude, vibration gradually reduces. The maximum displacement of 10th, 80th, 140th and 200th are $2.523235 \mathrm{~m}, 0.911102346 \mathrm{~m}, 1.270945958 \mathrm{~m}$ and $1.315001323 \mathrm{~m}$. It means that structural response or the energy of the xy plane goes down and then goes up. After RBS is superimposed, structural response and motion in xy plane increases slightly. Table 3 shows the detailed parameters.

Table 3. Amplitude and Angle of XY plane oscillation for condition 1

\begin{tabular}{c|c|c|c|c|c|c}
\hline Node & $\boldsymbol{A m p C a b}(\mathbf{m})$ & $\boldsymbol{A m p C s w}(\mathbf{m})$ & $\begin{array}{c}\boldsymbol{A m p} \text { difference } \\
\text { rate }\end{array}$ & $\boldsymbol{A n g C a b}\left({ }^{\circ}\right)$ & $\boldsymbol{A n g C s w}\left({ }^{\circ}\right)$ & $\begin{array}{c}\text { Ang difference } \\
\text { rate }\end{array}$ \\
\hline 10 & 2.52323500 & 2.52324300 & $0.000 \%$ & 0.91561700 & 0.9127021 & $-0.318 \%$ \\
80 & 0.911102346 & 0.911394511 & $0.032 \%$ & -40.149901 & -41.391644 & $3.093 \%$ \\
140 & 1.270945958 & 1.270957523 & $0.001 \%$ & -72.166466 & -72.166832 & $0.001 \%$ \\
200 & 1.315001323 & 1.315305591 & $0.023 \%$ & -81.537765 & -81.537482 & $0.000 \%$ \\
\hline
\end{tabular}

With depth increase, movement of structure in xy plane vibrates from the narrow amplitude oscillation in $\mathrm{x}$ direction gradually to the narrow amplitude oscillation in $\mathrm{y}$ direction. The amplitude oscillation of upper node decreases to a maximum near top suspension region 10th-80th. As water depth increases, amplitude of y-direction swing 
gradually increases, and the structural response or energy of xy plane decreases first and then rises.

The study above helps understand response of SCR structural particles at different positions from a space angle.

\section{Analysis of transverse direction of SCR response under linear TSM, RBS and WA.}

For riser, the above displacement research in xy plane is response in the SCR pipe body plane. This chapter mainly focuses on the response of $\mathrm{z}$ direction (transverse flow).

The displacement and its frequency in transverse direction is simulated from perspective normal to xy plane, which is non-negligible part of three-dimensional analysis. It is an important problem for structural vibration system study.

Cross flow response of structure including response caused by vortex-induced vibration (VIV), transverse direction action, RBS and so on is worth studying.

Compared with structural response caused by bending vibration and vortex-induced vibration, the response study of RBS in transverse direction is relatively few. This chapter focuses on the response characteristics in the transverse direction under linear WA, linear TSM and RBS.

Figure 4 shows the displacement of node 10-200th in condition 1.

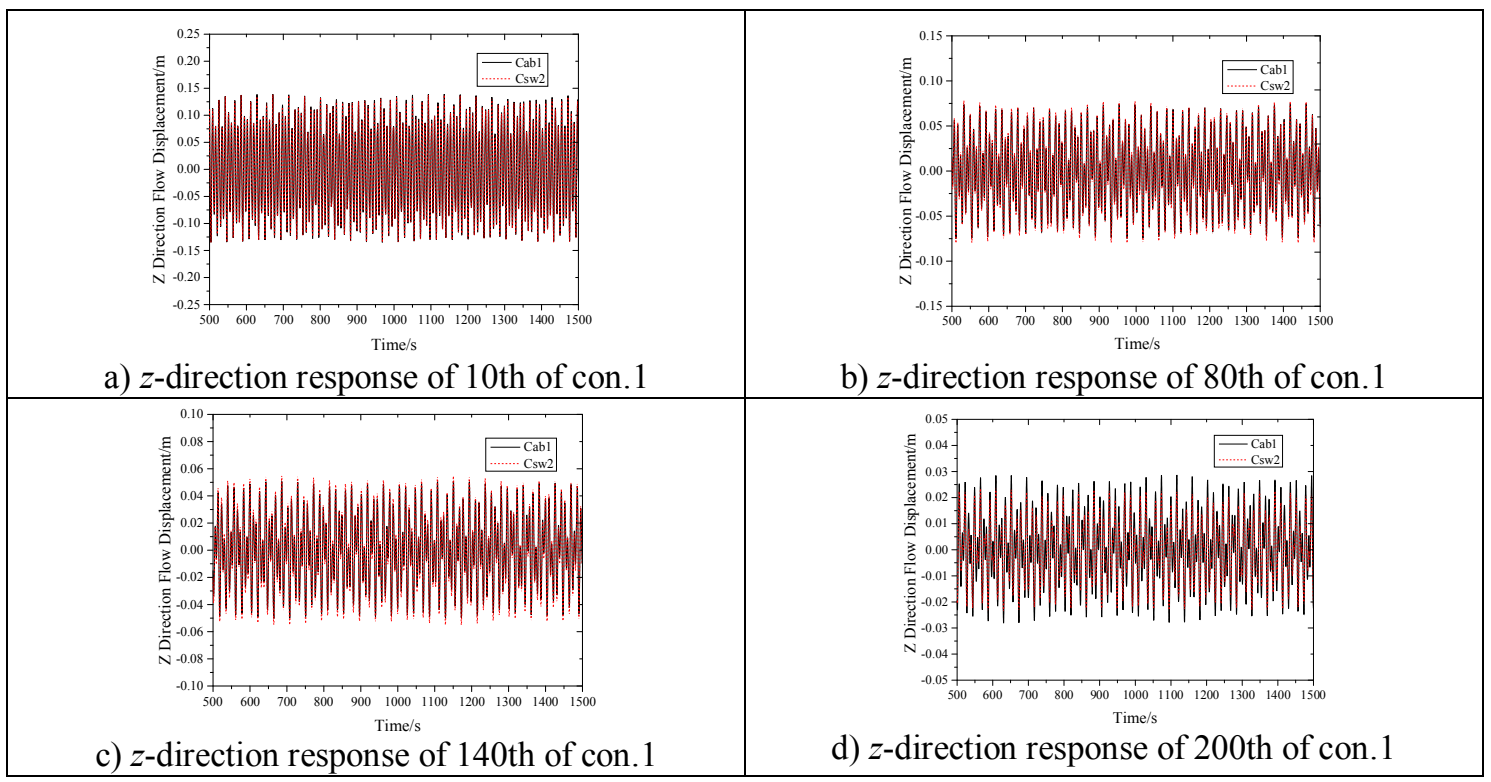

Figure 4. The transverse-direction response of 10th-200th of riser of con. 1

Figure 4 shows simulation results of con.1, respectively, and results of node 10th200th are random sequences. The maximum of response are $0.13922 \mathrm{~m}, 0.07491 \mathrm{~m}$, $0.0509 \mathrm{~m}$ and $0.0286 \mathrm{~m}$, and minimum of response are $-0.13483 \mathrm{~m},-0.07496 \mathrm{~m}$, $0.05014 \mathrm{~m}$ and $-0.02802 \mathrm{~m}$. Standard deviation of fluctuation are $0.07544,0.03754$, 0.02523 and 0.01304 . It shows that with node number increase, that is, the region away from top suspension point or water depth increase, cross flow response of riser is weakened. And Figure 6 shows it is under the action of WA and TSM. Not considering 
action of current, the WA on the structure and TSM both weaken with the depth increase, and then response weakens.

Under WA and TSM coupling RBS model, results of node 10th-200th are still random sequences. The maximum of the response are $0.1383 \mathrm{~m}, 0.0782 \mathrm{~m}, 0.0550 \mathrm{~m}$, and $0.0237 \mathrm{~m}$. And minimum of response are $-0.1350 \mathrm{~m},-0.08003 \mathrm{~m},-0.0551 \mathrm{~m}$ and $0.0237 \mathrm{~m}$. The standard deviation of fluctuation are $0.07534,0.03959,0.02733 .0 .01143$. It can be seen that as distance of region away from the top suspension point increases, transverse flow response of structure is weakened. Figure 6 shows the response under WA, TSM and RBS. It is noteworthy that when RBS is superimposed, response trend is equivalent to that only under WA and TSM. And effect of RBS on the riser does not exceed that of WA and TSM.

RBS influence with WA and TSM is briefly regarded as a linear overlay or superposition. Node 10-200th have a maximum increase of $-0.69 \%, 4.35 \%, 8.02 \%$ and $17.48 \%$. The minimum increases by $0.14 \%, 6.76 \%, 9.79 \%$ and $-15.45 \%$. With increase of water depth, RBS effect first augments and then reduces. That is, the trend is positively related to $\mathrm{S}$ which is RBS vector diameter, as shown in Figure 1. And this change is related to response of xy plane or the trend that energy decreasing and then increasing. It can be explained in terms of the total energy conservation of the structure. Other conditions have similar trends, as shown in Figures 5-6 and Tables 4-6.

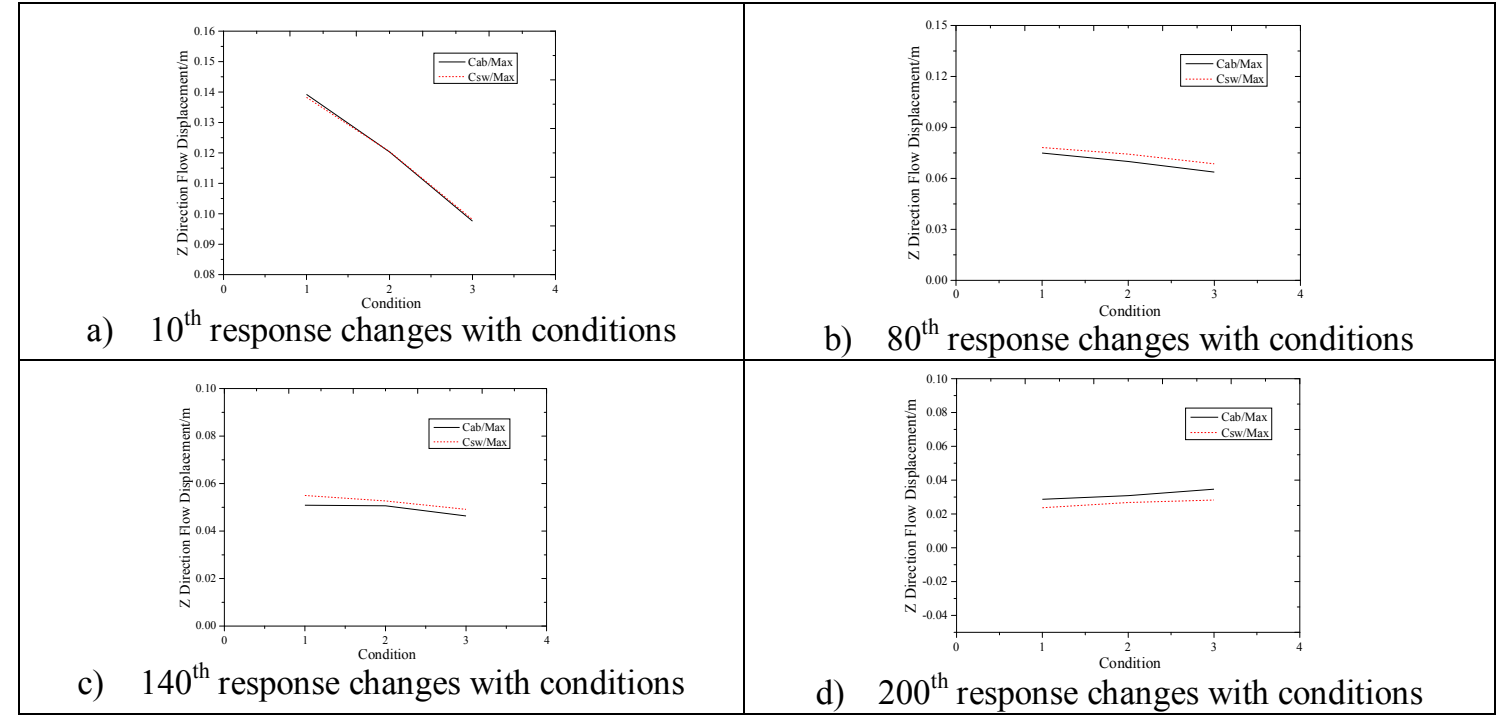

Figure 5. The structural response of 10th-200th changes with the conditions

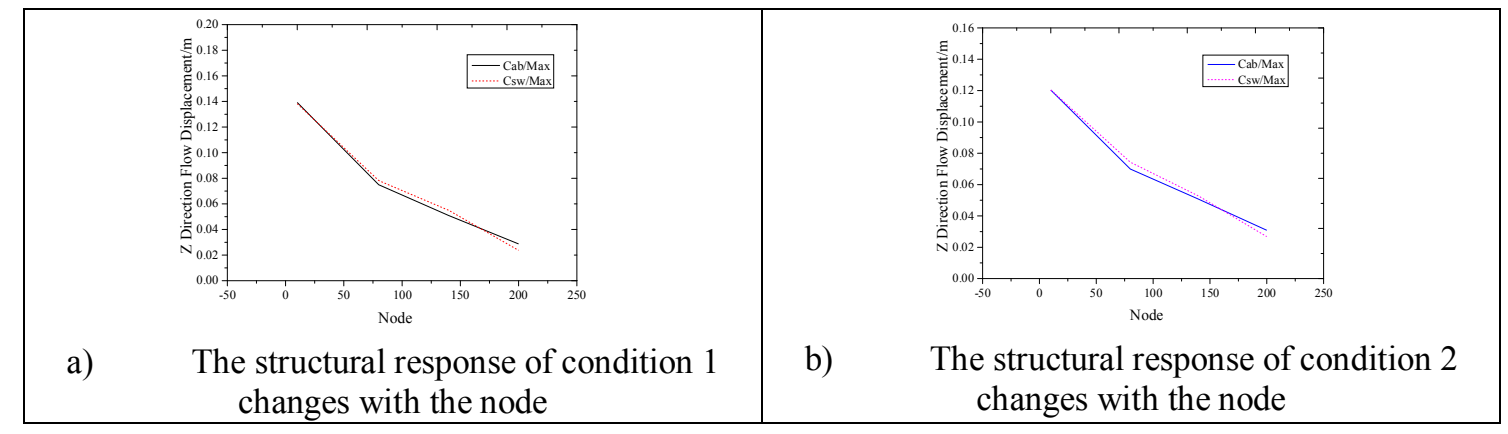




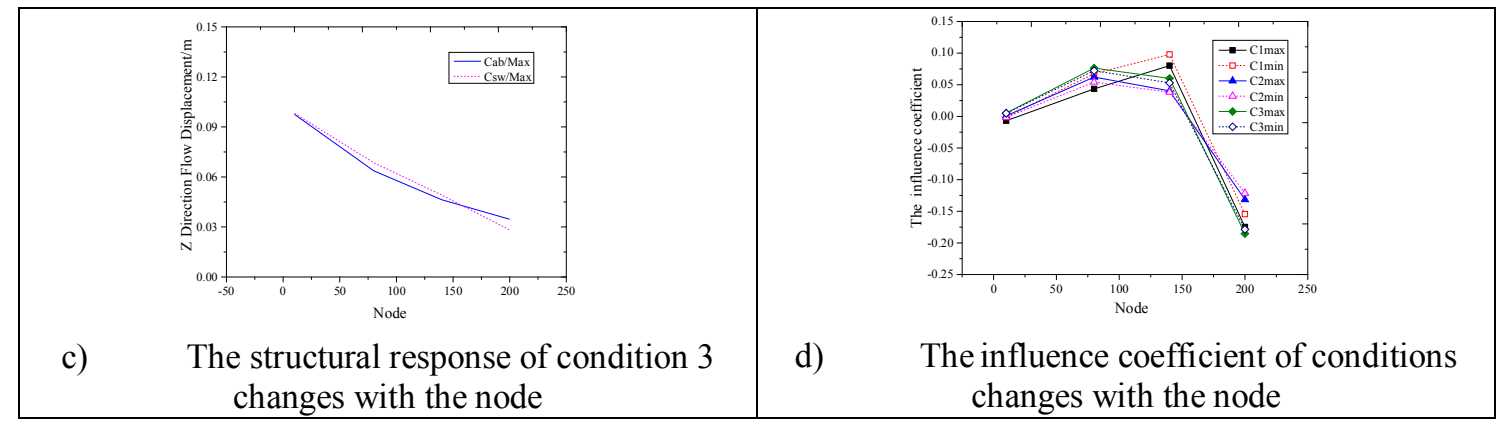

Figure 6. The structural response of condition changes with the node

Table 4. The $z$ direction maximum, minimum and equilibrium position of node displacement of each condition

\begin{tabular}{|c|c|c|c|c|c|c|c|c|c|}
\hline Cdt & Node & $\begin{array}{c}C a b_{\text {rmax }} \\
\text { (m) }\end{array}$ & $\begin{array}{c}C s w_{\text {rmax }} \\
\text { (m) }\end{array}$ & $I n c_{\text {rmax }}$ & $\begin{array}{c}C a b_{r m i n} \\
\text { (m) }\end{array}$ & $\begin{array}{l}C s w_{r m i n} \\
(\mathrm{~m})\end{array}$ & $I n c_{r m i n}$ & $\begin{array}{c}\boldsymbol{C a b}_{\text {rmean }} \\
\text { (m) }\end{array}$ & $\begin{array}{c}C S w_{\text {rmean }} \\
\text { (m) }\end{array}$ \\
\hline 1 & $10^{\text {th }}$ & 0.13922 & 0.13826 & $-0.69 \%$ & -0.13483 & -0.13502 & $0.14 \%$ & $4.28518 \mathrm{e}-4$ & $-3.21780 \mathrm{e}-4$ \\
\hline 1 & $80^{\text {th }}$ & 0.07491 & 0.07817 & $4.35 \%$ & -0.07496 & -0.08003 & $6.76 \%$ & $2.43481 \mathrm{e}-4$ & $-4.59409 \mathrm{e}-4$ \\
\hline 1 & $140^{\text {th }}$ & 0.0509 & 0.05498 & $8.02 \%$ & -0.05014 & -0.05505 & $9.79 \%$ & $2.97209 \mathrm{e}-4$ & $-3.32327 \mathrm{e}-4$ \\
\hline 1 & $200^{\text {th }}$ & 0.02866 & 0.02365 & $-17.48 \%$ & -0.02802 & -0.02369 & $-15.45 \%$ & $2.96427 \mathrm{e}-4$ & $-2.06679 \mathrm{e}-4$ \\
\hline 2 & $10^{\text {th }}$ & 0.12032 & 0.12043 & $0.09 \%$ & -0.11648 & -0.11631 & $-0.15 \%$ & $1.32305 \mathrm{E}-4$ & $5.31018 \mathrm{E}-4$ \\
\hline 2 & $80^{\text {th }}$ & 0.06994 & 0.07429 & $6.22 \%$ & -0.07190 & -0.0758 & $5.42 \%$ & $-9.64794 \mathrm{E}-5$ & $2.39313 \mathrm{E}-4$ \\
\hline 2 & $140^{\text {th }}$ & 0.05065 & 0.05269 & $4.03 \%$ & -0.05185 & -0.05383 & $3.82 \%$ & $-6.27087 \mathrm{E}-5$ & $2.25175 \mathrm{E}-4$ \\
\hline 2 & $200^{\text {th }}$ & 0.03085 & 0.02679 & $-13.16 \%$ & -0.03057 & -0.02686 & $-12.14 \%$ & $-3.37246 \mathrm{E}-5$ & $2.19485 \mathrm{E}-4$ \\
\hline 3 & $10^{\text {th }}$ & 0.0976 & 0.09811 & $0.52 \%$ & -0.09532 & -0.09579 & $0.49 \%$ & $7.800820 \mathrm{E}-5$ & $5.39977 \mathrm{E}-4$ \\
\hline 3 & $80^{\text {th }}$ & 0.06373 & 0.06859 & $7.63 \%$ & -0.06609 & -0.07087 & $7.23 \%$ & $-2.96270 \mathrm{E}-5$ & $3.63584 \mathrm{E}-4$ \\
\hline 3 & $140^{\text {th }}$ & 0.04637 & 0.04915 & $6.00 \%$ & -0.04812 & -0.05067 & $5.30 \%$ & $-3.91931 \mathrm{E}-5$ & $2.84196 \mathrm{E}-4$ \\
\hline 3 & $200^{\text {th }}$ & 0.03464 & 0.02822 & $-18.53 \%$ & -0.03573 & -0.02935 & $-17.86 \%$ & $-9.64304 \mathrm{E}-6$ & $2.50152 \mathrm{E}-4$ \\
\hline
\end{tabular}

Table 5. $z$ direction fluctuation standard deviation reduction and maximum normalized coefficient of each condition

\begin{tabular}{|c|c|c|c|c|c|c|c|c|c|}
\hline$C d t$ & Node & $\boldsymbol{C a b}_{r s t d}$ & $\boldsymbol{R} d t_{r c a b}$ & $C \boldsymbol{s} w_{r s t d}$ & $\boldsymbol{R d t}_{r c s w}$ & $\boldsymbol{C a} \boldsymbol{b}_{r \max }$ & $N m l_{\text {rmax }}$ & $\boldsymbol{C} \boldsymbol{s} \boldsymbol{w}_{r \max }$ & $N m l_{r m i n}$ \\
\hline 1 & $10^{\text {th }}$ & 0.07544 & $50.24 \%$ & 0.07534 & $47.45 \%$ & 0.13922 & 1.00 & 0.13826 & 1.00 \\
\hline 1 & $80^{\text {th }}$ & 0.03754 & $16.32 \%$ & 0.03959 & $16.27 \%$ & 0.12032 & 0.86 & 0.12043 & 0.87 \\
\hline 1 & $140^{\text {th }}$ & 0.02523 & $16.16 \%$ & 0.02733 & $21.10 \%$ & 0.0976 & 0.70 & 0.09811 & 0.71 \\
\hline 1 & $200^{\text {th }}$ & 0.01304 & $17.29 \%$ & 0.01143 & $15.17 \%$ & 0.07491 & 1.00 & 0.07817 & 1.00 \\
\hline 2 & $10^{\text {th }}$ & 0.06803 & $46.38 \%$ & 0.06808 & $42.60 \%$ & 0.06994 & 0.93 & 0.07429 & 0.95 \\
\hline 2 & $80^{\text {th }}$ & 0.03648 & $15.21 \%$ & 0.03908 & $17.19 \%$ & 0.06373 & 0.85 & 0.06859 & 0.88 \\
\hline 2 & $140^{\text {th }}$ & 0.02613 & $16.10 \%$ & 0.02738 & $21.81 \%$ & 0.0509 & 1.00 & 0.05498 & 1.00 \\
\hline 2 & $200^{\text {th }}$ & 0.01518 & $22.31 \%$ & 0.01253 & $18.40 \%$ & 0.05065 & 1.00 & 0.05269 & 0.96 \\
\hline 3 & $10^{\text {th }}$ & 0.06039 & $38.05 \%$ & 0.06079 & $33.90 \%$ & 0.04637 & 0.91 & 0.04915 & 0.89 \\
\hline 3 & $80^{\text {th }}$ & 0.03741 & $20.77 \%$ & 0.04018 & $22.57 \%$ & 0.02866 & 1.00 & 0.02365 & 1.00 \\
\hline 3 & $140^{\text {th }}$ & 0.02487 & $13.51 \%$ & 0.02646 & $22.77 \%$ & 0.03085 & 1.08 & 0.02679 & 1.13 \\
\hline 3 & $200^{\text {th }}$ & 0.01671 & $27.67 \%$ & 0.01262 & $20.76 \%$ & 0.03464 & 1.21 & 0.02822 & 1.19 \\
\hline
\end{tabular}

$\mathrm{Cab}$ represents the motion response under WA and TSM. Csw is a superposition of the motion of WA, TSM and RBS 
Table 6. Calculation of reduction of each node response compared to condition 1

\begin{tabular}{|c|c|c|c|c|c|c|c|c|c|}
\hline Cdt & Node & $\begin{array}{c}C a b_{\text {rmax }} \\
\text { (m) }\end{array}$ & $\boldsymbol{R} d t_{r \max }$ & $\begin{array}{c}C s w_{r \max } \\
\text { (m) }\end{array}$ & $\boldsymbol{R} d t_{r \max }$ & $\begin{array}{c}C a b_{\text {rmin }} \\
\text { (m) }\end{array}$ & $\boldsymbol{R} d t_{r m i n}$ & $\begin{array}{c}\boldsymbol{C s w}_{r \min } \\
\text { (m) }\end{array}$ & $\boldsymbol{R} d t_{r \min }$ \\
\hline 1 & $10^{\text {th }}$ & 0.13922 & $46.19 \%$ & 0.13826 & $43.46 \%$ & -0.13483 & $44.40 \%$ & -0.13502 & $40.73 \%$ \\
\hline 1 & $80^{\text {th }}$ & 0.07491 & $17.25 \%$ & 0.07817 & $16.77 \%$ & -0.07496 & $18.41 \%$ & -0.08003 & $18.50 \%$ \\
\hline 1 & $140^{\text {th }}$ & 0.0509 & $15.97 \%$ & 0.05498 & $22.66 \%$ & -0.05014 & $16.41 \%$ & -0.05505 & $23.23 \%$ \\
\hline 1 & $200^{\text {th }}$ & 0.02866 & $20.59 \%$ & 0.02365 & $17.11 \%$ & -0.02802 & $20.78 \%$ & -0.02369 & $17.55 \%$ \\
\hline 2 & $10^{\text {th }}$ & 0.12032 & $41.87 \%$ & 0.12043 & $38.31 \%$ & -0.11648 & $38.27 \%$ & -0.11631 & $34.83 \%$ \\
\hline 2 & $80^{\text {th }}$ & 0.06994 & $16.03 \%$ & 0.07429 & $17.94 \%$ & -0.0719 & $17.21 \%$ & -0.0758 & $18.89 \%$ \\
\hline 2 & $140^{\text {th }}$ & 0.05065 & $16.46 \%$ & 0.05269 & $21.51 \%$ & -0.05185 & $18.27 \%$ & -0.05383 & $23.19 \%$ \\
\hline 2 & $200^{\text {th }}$ & 0.03085 & $25.64 \%$ & 0.02679 & $22.25 \%$ & -0.03057 & $26.24 \%$ & -0.02686 & $23.09 \%$ \\
\hline 3 & $10^{\text {th }}$ & 0.0976 & $34.70 \%$ & 0.09811 & $30.09 \%$ & -0.09532 & $30.67 \%$ & -0.09579 & $26.02 \%$ \\
\hline 3 & $80^{\text {th }}$ & 0.06373 & $17.79 \%$ & 0.06859 & $19.81 \%$ & -0.06609 & $18.85 \%$ & -0.07087 & $21.09 \%$ \\
\hline 3 & $140^{\text {th }}$ & 0.04637 & $12.02 \%$ & 0.04915 & $21.33 \%$ & -0.04812 & $13.00 \%$ & -0.05067 & $22.26 \%$ \\
\hline 3 & $200^{\text {th }}$ & 0.03464 & $35.49 \%$ & 0.02822 & $28.76 \%$ & -0.03573 & $37.48 \%$ & -0.02935 & $30.64 \%$ \\
\hline
\end{tabular}

With the increase of nodes, the attenuation of maximum for node 10th, 80th, 140th and 200 th are $46.19 \%, 17.25 \%, 15.97 \%$ and $20.59 \%$. The attenuation of minimum are $44.40 \%, 18.41 \%, 16.41 \%$ and $20.78 \%$. And the attenuation of standard deviation are $50.24 \%, 16.32 \%, 16.16 \%$ and $17.29 \%$. With water depth increase, maximum, minimum and standard deviation of structure attenuate the most in top suspension point region, followed by touch down point region. The structural response decays to zero gradually, and attenuation amplitude in the intermediate region of riser is small. It is related to decrease of WA and TSM on the structure, with increase of depth. SCR is gradually close to the seabed, and finally presents as the submarine streamline section. The structure is subject to the reaction force of seabed support, which is more obvious in the bottom contact area. In middle region, TSM is related to the attenuation of WA and increasing of RBS, and the reduction shows a small attenuation.

With the increase of conditions, the attenuation of node 10th maximum are $46.19 \%$, $41.87 \%$, and $34.70 \%$. The attenuation of minimum are $44.40 \%, 38.27 \%$ and $30.67 \%$. The attenuation of standard deviation are $50.24 \%, 46.38 \%$ and $38.05 \%$. As the condition increases, the attenuation of maximum, minimum and standard deviation gradually decreases. And the structural attenuation is more affected by the amplitude of condition of TSM as shown in Table 2, showing a positive correlation.

The rest of the nodes are shown in Tables 5-6. It is noteworthy that after RBS superimposed, the attenuation tendency of response is equivalent to that only under WA and TSM. And the effect of RBS on riser does not exceed that of WA and TSM.

Node response of 10-200th are compared in Figures 5-6. With motion amplitude decrease, each node response decreases or increases in different location. On the whole, the hanging point 10th have a more obvious decrease with conditions, and the response have an increase near the touch down point. The maximum influence coefficient of structure is about 20\%, as shown in Figure 6 and Table 4.

\section{Spectrum analysis}

For z-response, spectrum analysis can transform the random signal in time domain into the signal in the frequency domain. Spectrum analysis can obtain main frequency 
of structure. It shows that there is a large value at a certain frequency point, and there is a maximum at one or more frequencies. By extracting maximum, this chapter mainly analyzes it:

1. Relative attenuation with the increase of water depth;

2. Increase or decrease of frequency caused by RBS;

3. The variation characteristics of structural displacement response frequency with the TSM frequency.

Through spectrum analysis, displacement spectrum responses of 10th, 80th, 140th and 200th are obtained, as shown in Figure 7.

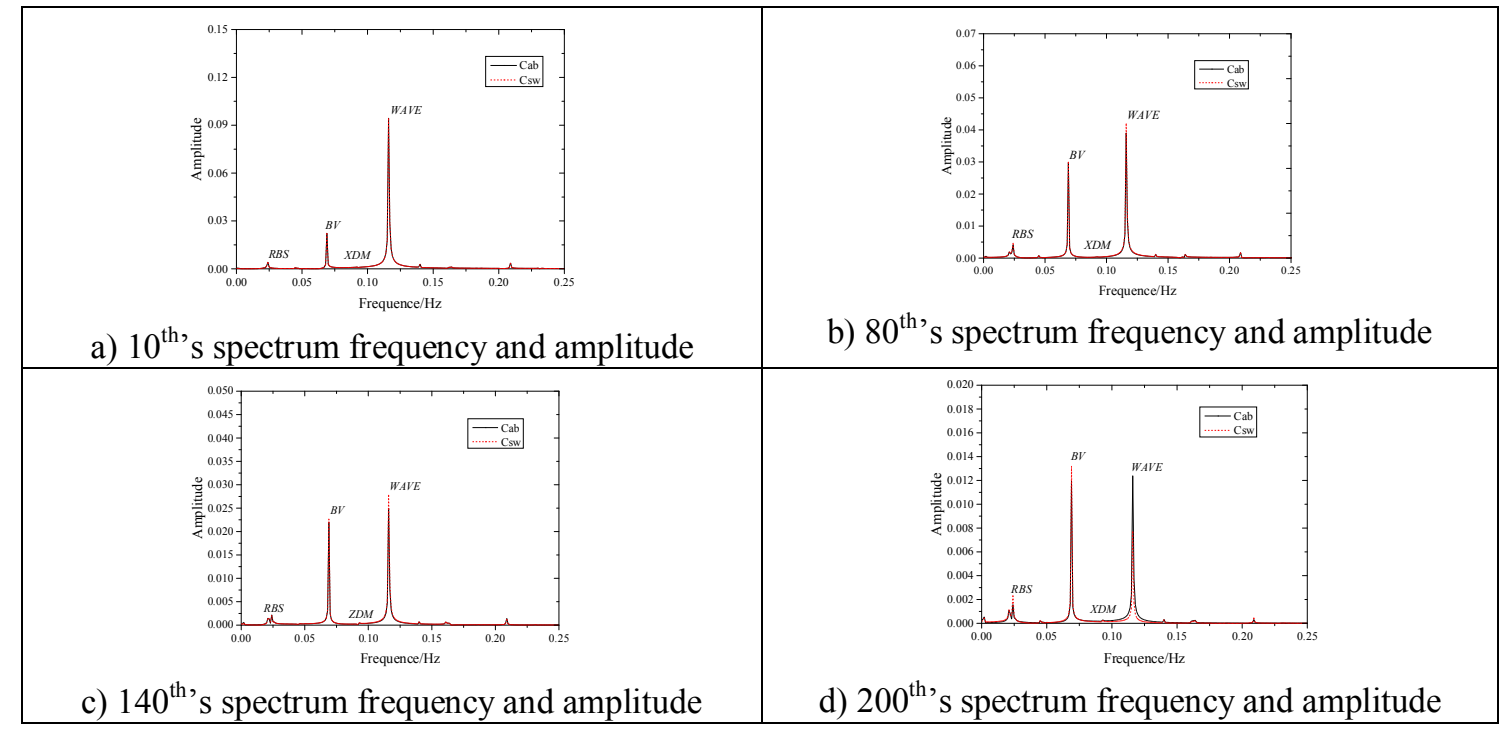

Figure 7. 10-200 ${ }^{\text {th }}$ s spectrum amplitude of con.1

Figure 7 shows displacement spectrum response of condition 1's node 10-200th. The $\mathrm{x}$ value is $0.115988 \mathrm{~Hz}$, which is very close to WA frequency $0.11622 \mathrm{~Hz}$. It could be considered that the structure's frequency is the same as that of the applied external load, and it is a forced motion.

For y value (spectral amplitude), as the node increases, amplitude values of each node are shown in Table 7. Spectrum response of 10th, 80th, 140th and 200th are $0.094425,0.039063,0.025002$ and 0.012396 . The amplitude of node is decreasing, indicating that maximum energy point of structure is decreasing, as shown in Table 7. This point can be proved from response of z-direction displacement of the structure, as shown in Table 4. For Table 7, it is similar to the corresponding change of displacement in terms of main frequency first increasing and then decreasing with increase of node. In contrast to change of energy or amplitude in xy plane, main frequency and displacement in $\mathrm{z}$ direction can be explained from energy conservation in three directions. With the decrease in the xy plane before the increase, the $\mathrm{z}$ direction displacement due to RBS in the $3 \mathrm{~d}$ direction presents an opposite change, as shown in Table 4.

From the calculation of the same node in different conditions, the values of node 10th in condition 1-3 without RBS are $0.094425 \mathrm{~m}, 0.086288 \mathrm{~m}$ and $0.077539 \mathrm{~m}$. And considering the RBS, the values are $0.094317 \mathrm{~m}, 0.086327 \mathrm{~m}$ and $0.078034 \mathrm{~m}$. Relatively speaking, the difference between the RBS and non-RBS are $-0.11 \%, 0.05 \%$ 
and $0.64 \%$. It indicates that main frequency of the structure increases or decreases after the RBS is applied. The maximum influence for 10 th is $0.64 \%$. At nodes 80 th, 140th and 200th, the main frequency of the structure increases by $8.17 \%, 11.27 \%$ and $37.71 \%$. It indicates that when RBS applied, main frequency of riser changes. It increases above 140th or in non-touch down area, and decreases in touch down area, as shown in Tables 7-8.

Table 7. The maximum value table of non-RBS and RBS spectrum

\begin{tabular}{c|c|c|c|c|c|c|c}
\hline $\boldsymbol{C} \boldsymbol{d} \boldsymbol{t}$ & Node & $\boldsymbol{X} \boldsymbol{r}(\mathbf{H z})$ & $\boldsymbol{C a b} / \boldsymbol{Y r}$ & $\boldsymbol{R d t}_{\boldsymbol{r} \text { cab }}$ & $\boldsymbol{X r} \mathbf{( H z )}$ & $\boldsymbol{C s w} / \boldsymbol{Y r}$ & $\boldsymbol{R d t}_{\boldsymbol{r c s w}}$ \\
\hline 1 & $10^{\text {th }}$ & 0.115988 & 0.094425 & $58.63 \%$ & 0.115988 & 0.094317 & $55.52 \%$ \\
1 & $80^{\text {th }}$ & 0.115988 & 0.039063 & $14.89 \%$ & 0.115988 & 0.041956 & $14.99 \%$ \\
1 & $140^{\text {th }}$ & 0.115988 & 0.025002 & $13.35 \%$ & 0.115988 & 0.027820 & $21.31 \%$ \\
1 & $200^{\text {th }}$ & 0.115988 & 0.012396 & $13.13 \%$ & 0.115988 & 0.007721 & $8.19 \%$ \\
2 & $10^{\text {th }}$ & 0.115988 & 0.086288 & $51.42 \%$ & 0.115988 & 0.086327 & $47.48 \%$ \\
2 & $80^{\text {th }}$ & 0.115988 & 0.041916 & $15.99 \%$ & 0.115988 & 0.045341 & $17.64 \%$ \\
2 & $140^{\text {th }}$ & 0.115988 & 0.028117 & $13.18 \%$ & 0.115988 & 0.030113 & $21.88 \%$ \\
2 & $200^{\text {th }}$ & 0.115988 & 0.016743 & $19.40 \%$ & 0.115988 & 0.011225 & $13.00 \%$ \\
3 & $10^{\text {th }}$ & 0.115988 & 0.077539 & $39.19 \%$ & 0.115988 & 0.078034 & $35.16 \%$ \\
3 & $80^{\text {th }}$ & 0.115988 & 0.047149 & $22.04 \%$ & 0.115988 & 0.050597 & $23.30 \%$ \\
3 & $140^{\text {th }}$ & 0.115988 & 0.030059 & $13.88 \%$ & 0.115988 & 0.032412 & $23.62 \%$ \\
3 & 200 th & 0.115988 & 0.019296 & $24.89 \%$ & 0.115988 & 0.013983 & $17.92 \%$ \\
\hline
\end{tabular}

Table 8. The spectrum analysis and normalized table of nodes under different conditions

\begin{tabular}{c|c|c|c|c|c|c|c|c|c}
\hline $\boldsymbol{C} \boldsymbol{d} t$ & Node & $\boldsymbol{X} \boldsymbol{r}(\mathbf{H z})$ & $\mathbf{C a b} / \mathbf{Y} \mathbf{r}$ & $\mathbf{C s w} / \mathbf{Y r}$ & $\mathbf{I n c r}$ & $\mathbf{C d t r}$ & $\mathbf{N o d e}^{\text {th }}$ & $\boldsymbol{N m \boldsymbol { ~ }}_{\boldsymbol{r m a x}}$ & $\boldsymbol{N m \boldsymbol { l }}_{\boldsymbol{r m i n}}$ \\
\hline 1 & $10^{\text {th }}$ & 0.115988 & 0.094425 & 0.094317 & $-0.11 \%$ & 1 & $10^{\text {th }}$ & 1.00 & 1.00 \\
1 & $80^{\text {th }}$ & 0.115988 & 0.039063 & 0.041956 & $7.41 \%$ & 2 & $10^{\text {th }}$ & 0.91 & 0.92 \\
1 & $140^{\text {th }}$ & 0.115988 & 0.025002 & 0.027820 & $11.27 \%$ & 3 & $10^{\text {th }}$ & 0.82 & 0.83 \\
1 & $200^{\text {th }}$ & 0.115988 & 0.012396 & 0.007721 & $-37.71 \%$ & 1 & $80^{\text {th }}$ & 1.00 & 1.00 \\
2 & $10^{\text {th }}$ & 0.115988 & 0.086288 & 0.086327 & $0.05 \%$ & 2 & $80^{\text {th }}$ & 1.07 & 1.08 \\
2 & $80^{\text {th }}$ & 0.115988 & 0.041916 & 0.045341 & $8.17 \%$ & 3 & $80^{\text {th }}$ & 1.21 & 1.21 \\
2 & $140^{\text {th }}$ & 0.115988 & 0.028117 & 0.030113 & $7.10 \%$ & 1 & $140^{\text {th }}$ & 1.00 & 1.00 \\
2 & $200^{\text {th }}$ & 0.115988 & 0.016743 & 0.011225 & $-32.96 \%$ & 2 & $140^{\text {th }}$ & 1.12 & 1.08 \\
3 & $10^{\text {th }}$ & 0.115988 & 0.077539 & 0.078034 & $0.64 \%$ & 3 & $140^{\text {th }}$ & 1.20 & 1.17 \\
3 & $80^{\text {th }}$ & 0.115988 & 0.047149 & 0.050597 & $7.31 \%$ & 1 & $200^{\text {th }}$ & 1.00 & 1.00 \\
3 & $140^{\text {th }}$ & 0.115988 & 0.030059 & 0.032412 & $7.83 \%$ & 2 & $200^{\text {th }}$ & 1.35 & 1.45 \\
3 & $200^{\text {th }}$ & 0.115988 & 0.019296 & 0.013983 & $-27.53 \%$ & 3 & $200^{\text {th }}$ & 1.56 & 1.81 \\
\hline
\end{tabular}

At the same time, with the increase of conditions, the main frequency of 10 th decreases. The other nodes show an increase of main frequency, with an increase of $80 \%$ in the bottom touch down area. The energy goes down at 10th, and the amplitude goes down, while it goes up in other areas, with amplitude shown in Table 8. After the normalized operation, coefficient in touch down point without RBS are 1.00, 1.35 and 1.56. And the coefficient superposed RBS are 1.00, 1.45 and 1.81. The coefficients of the two groups differ greatly, and the differences in other conditions are not significant. 
In addition, it indicates that $\mathrm{Z}$ direction response of each position or node is proportional to the frequency of load applied. There is a certain resonance between loads and the response increases.

It is worth that Table 9 gives some data of wave main frequency influence. Figure 8 shows the effect of RBS on spectrum amplitude of different loads of the structure. These data can make some judgments about the effects of different loads. From 10th to 200th, the wave main frequency has an influence of $77.62 \%, 53.31 \%, 50.32 \%$, and $47.35 \%$. With the increase of water depth, the influence gradually decreases. RBS increases the spectral analysis amplitude of structural RBS, bending vibration, TSM and WA. The RBS increases response of riser to different degrees. It is worth noting that the bottom contact point is $200^{\text {th }}$. And RBS reduces both WA response and TSM response, as shown in Figure 8. To some extent, WA and TSM reduce oscillation of RBS at bottom TDP. It is similar to decrease of riser displacement in Table 4. In a word, spectrum analysis is of great significance for structural response interpretation.

Table 9. The spectrum analysis and normalized table of nodes under different conditions

\begin{tabular}{|c|c|c|c|c|c|c|c|c|c|c|}
\hline \multicolumn{2}{|c|}{ Conditions } & $\begin{array}{c}R B R / x \\
(\mathrm{~Hz})\end{array}$ & $R B R / y$ & $\begin{array}{l}B V / x \\
(\mathrm{~Hz})\end{array}$ & $B V / y$ & $\begin{array}{c}X D M / x \\
(\mathrm{~Hz})\end{array}$ & $X D M / y$ & $\begin{array}{c}W A V E / x \\
(\mathrm{~Hz})\end{array}$ & WAVE/y & \begin{tabular}{|c|}
$\begin{array}{c}\text { WAVE } \\
\text { influence }\end{array}$ \\
\end{tabular} \\
\hline \multicolumn{2}{|c|}{$\begin{array}{c}\text { Theoretical } \\
\text { (Yao et al., 2018) }\end{array}$} & 029 & I & 0.0640 & I & 0.0930 & I & 0.1162 & I & \\
\hline \multirow{2}{*}{$10^{\text {th }}$} & $\mathrm{Cab}$ & 0.0240 & 0.0041 & 0.0690 & 0.0223 & 0.0930 & $8.1679 \mathrm{E}-4$ & 0.1160 & 0.0944 & \\
\hline & & 0240 & 0.0041 & 0.0690 & 0.0222 & 0.0930 & 8.0093E-4 & 0.1160 & & $77.68 \%$ \\
\hline \multirow{2}{*}{$80^{\text {th }}$} & & 0.0240 & 0.0041 & 0.0690 & 0.0298 & 0.0930 & $3.4824 \mathrm{E}-4$ & 0.1160 & 0.0391 & $53.31 \%$ \\
\hline & Csw & 0.0240 & 0.0047 & 0.0690 & 0.0300 & 0.0930 & 3.7593E-4 & 0.1160 & 0.0420 & $54.49 \%$ \\
\hline \multirow{2}{*}{$140^{\text {th }}$} & & 0.0240 & 0.0022 & 0.0690 & 0.0220 & 0.0930 & $4.8580 \mathrm{E}-4$ & 0.1160 & 0.0250 & $50.32 \%$ \\
\hline & $\mathrm{Csw}$ & 0.0240 & & 0.0690 & 0.0227 & 0.0930 & $5.2845 \mathrm{E}-4$ & 0.1160 & & $52.33 \%$ \\
\hline \multirow{2}{*}{$200^{\text {th }}$} & & 0.0240 & 0.0016 & 0.0690 & 0.0119 & 0.0930 & 2.9034E-4 & 0.1160 & 0.0124 & $47.35 \%$ \\
\hline & Csw & 0.0240 & 0.0023 & 0.0690 & 0.0132 & 0.0930 & $2.5167 \mathrm{E}-4$ & 0.1160 & 0.0077 & $32.83 \%$ \\
\hline
\end{tabular}

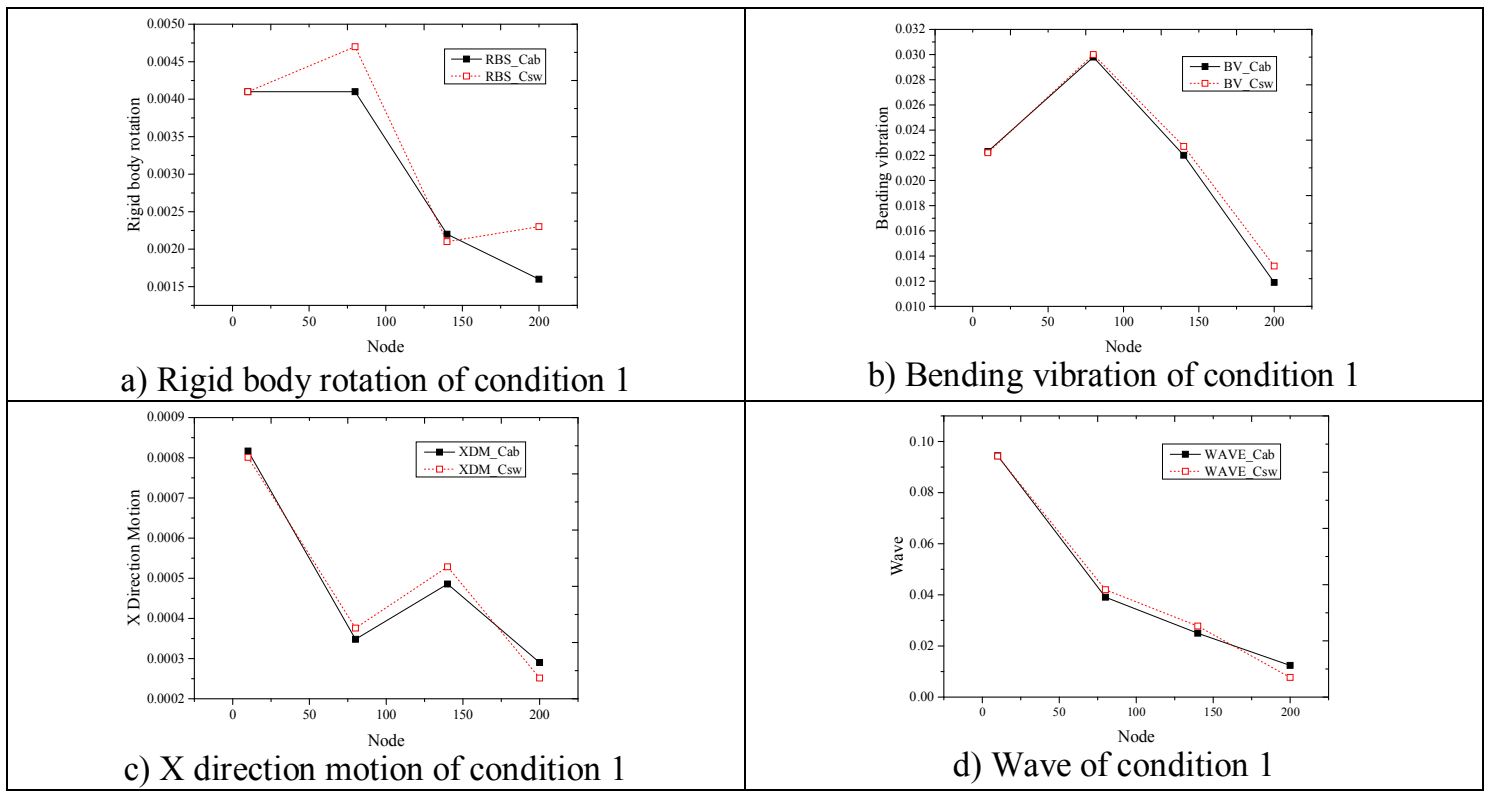

Figure 8. The effects of RBS on the amplitude of load frequency spectrum along water depth of condition 1 


\section{Conclusion}

It is a relatively important problem to simulate the transverse response of riser under RBS. The swing is around the rotating axis with TSP and TDP under WA. In this paper, without considering current, the relatively complicated response of riser coupled RBS is studied. And response is influenced by linear WA and TSM on the structure.

From a perspective of the overall motion pattern, response on the xy plane has developed from a narrow range swing in $\mathrm{x}$ direction to a narrow range oscillation in $\mathrm{y}$ direction. Under the WA, response of riser reduces gradually with depth. When coupling $\mathrm{x}$ direction TSM, phenomenon that response decreases when water depth increases still persists. In terms of variation in the depth direction of the structure, with increase of water depth, displacement decreases. And attenuation range is more intense in the top suspension region, while attenuation is weak in bottom region.

From the frequency of structure, it can be considered that the response of structure is the same as that of external load, and the response is forced motion. In terms of the conditions, as the node increases or the water depth increases, the main frequency first increases and then decreases, which is similar to the change of the structural response. In contrast to the change of energy or amplitude of response in the xy plane, it can be explained from the energy conservation of the structure in three directions of the node. As the response in the xy plane decreases first and then increases, the $\mathrm{z}$ direction response of RBS presents an opposite change.

After RBS is applied to structure, the main frequency increases above 140th or in the non-touch down area, and decreases in the touch down area. And the z-direction response of structure is inversely proportional to frequency increase of load applied and proportional to amplitude reduction.

Recommendations for future studies include the following points:

1) For the load term of the structural vibration equation, it is also necessary to consider the internal flow, isolated wave, etc. And multiple coupling calculation is needed to expand the program development and response calculation range.

2) To be specific, in this paper, linear wave is adopted, and more random wave check is required. Fluid-solid coupling phenomenon also needs to be taken into consideration. And it is suggested to adopt wake oscillator programming for the further calculation.

3 ) It is necessary to study response from a three-dimensional perspective, and spatial response characteristics cannot be easily ignored.

4) This paper considers contribution of load to response from the perspective of frequency amplitude. And it needs more detailed and accurate calculation from the contribution of load response.

5) In this paper, the number of conditions is few and it is suggested to search for more conditions to evaluate the effect of RBS by a safety ratio.

6) In this paper, there is also a deficiency in analytical calculation of structural vibration. It is suggested to increase the analytical solution of RBS, such as multi-load vibration matrix for verification, which may further increase the solution speed.

For analysis in Cable3D, the prospects are slightly better. It is expected that work of this paper could give some simulation advice for RBS research.

Acknowledgements. Thanks to all the help. And the work is supported by National natural science foundation of China (Number: 51239008, 51739010, 51679223, 51409232) and Natural science foundation of SHANGDONG province (ZR2016GM06). Thanks to National high-tech research and 
development program (863) (2013AA09A218) and National Science and Technology Major Project (2011ZX05026-005-003). Thanks to professor ZHANG Jun researching in Texas A\&M United States.

\section{REFERENCES}

[1] Bai, X. L. (2009): A study on Method for Nonlinear Analysis of Deepwater SCR Based on Inertial Coupling. - Ocean University of China, Qingdao.

[2] Bai, X. L., Huang, W. P., Murilo, A. V., Yang, C. F., Duan, M. L. (2015): Riser-soil interaction model effects on the dynamic behavior of a steel catenary riser. - Marine Structures 41: 53-76.

[3] Chang, S., Huang, W. P., Yang, C. F. (2017): The influence of nonlinear seabed on the dynamic response and fatigue damage of SCR at TDZ. - The Ocean Engineering 35(2): 67-82.

[4] Chen, X. H. (2002): Studies on Dynamic Interaction between Deep-Water Floating Structures and Their Mooring/Tendon Systems. - Texas A\&M University, College Station.

[5] Du, J. F. (2016): Investigation on Dynamic Response Characteristics and Fatigue Assessment Damage Methods for Mooring Lines of Semi-Submersible Platforms. Ocean University of China, Qingdao.

[6] Guan, D., Hsieh, S. C., Chiew, Y. M., Low, Y. M. (2019): Experimental study of scour around a forced vibrating pipeline in quiescent water. - Coastal Engineering 143: 1-11.

[7] He, Y. Y., Vaz, M. A., Caire, M. (2019): An inverse problem methodology for multiple parameter estimation in bend stiffeners. - Applied Ocean Research 83: 37-47.

[8] Kim, H. T., O'Reilly, O. M. (2019): Instability of catenary-type flexible risers conveying fluid in subsea environments. - Ocean Engineering 173: 98-115.

[9] Liang, N., Huang, W. P., Yang, C. F. (2016): Study on method of interaction between steel catenary riser and nonlinear seabed. - Ocean Engineering 34(1): 40-49.

[10] Lin, K., Wang, J. S. (2019): Numerical simulation of vortex-induced vibration of long flexible risers using a SDVM-FEM coupled method. - Ocean Engineering: 468-486.

[11] Liu, J. (2013): Study of Out-of-Plane Motion of SCRs with Rigid Swing. - Ocean University of China, Qingdao.

[12] Liu, J., Huang, W. P. (2013): A Nonlinear vortex induced vibration model of marine risers. - Journal of Ocean University of China 12(1): 32-36.

[13] Liu, J., Huang, W. P. (2014a): The coupling VIV analysis of SCRs with rigid swing. Journal of Ocean University of China 14: 645-650.

[14] Liu, J., Huang, W. P. (2014b): Fluid-structure interaction analysis for VIV of steel catenary risers. - Journal of Vibration and Shock 33(3): 41-45.

[15] Liu, J., Huang, W. P. (2014c): Analysis of out-of-plane motion of SCRS with rigid rotation. - Engineering Mechanic 31(2): 53-57.

[16] Lotsberg, I. (2019): Development of fatigue design standards for marine structures. Journal of Offshore Mechanics and Arctic Engineering 141(3). DOI: 10.1115/OMAE2017-62516.

[17] Numkam, G. L., Akbari, B. (2019): Effect of surfactant chemistry on drilling mud performance. - Journal of Petroleum Science and Engineering. DOI: 10.1016/j.petrol.2018.11.075

[18] Wang, W., Pei, X. J. (2019): An analytical structural strain method for steel umbilical in low cycle fatigue. - Journal of Offshore Mechanics and Arctic Engineering 141(1). DOI: 10.1115/1.4040721.

[19] Wu, J., Lekkala, M. R., Ong, M. C., Passano, E., Voie, P. E. (2019): Prediction of c ombined inline and crossflow vortex-induced vibrations response of deepwater risers. Journal of Offshore Mechanics and Arctic Engineering 141(4). DOI: 10.1115/1.4042072. 
[20] Yamamoto, M., Morooka, C. K. (2019): Feedback control system for blow-out preventer positioning. - Applied Ocean Research 82: 362-369.

[21] Yang, C. F. (2014): Study on Nonlinear Interaction between SCR and Seabed. - Ocean University of China, Quingdao.

[22] Yao, X. L., Huang, W. P., Liu, J., Luo, K. H. (2017): Study on the Coupling of Rigid swing and vortex-induced vibration of steel catenary risers. - Ship \& Ocean Engineering 46: 191-197.

[23] Yao, X. L., Huang, W. P., Chang, S., Liu, J., Fu, X. P. (2018): Modal analysis and test verification for out-of-plane vortex induced vibration of a SCR. - Journal of Vibration and Shock 37(13): 78-84.

[24] Zhao, X. W., van der Heijden, G. H. M. (2019): Dynamic torsional buckling: prebuckling waves and delayed instability. - Journal of Petroleum Science and Engineering 69: 13091320.

[25] Zhou, Y., Yang, C. F., Huang, W. P. (2017): Dynamic response analysis of steel catenary riser based on nonlinear stiffness of seabed. - Journal of Harbin Engineering University 38(3): 356-362. 